\title{
Divergence-free turbulence inflow conditions for large-eddy simulations with incompressible flow solvers
}

\author{
Yusik Kim, Ian P. Castro, Zheng-Tong Xie* \\ Faculty of Engineering and the Environment,University of Southampton, SO17 1BJ, \\ Southampton, UK
}

\begin{abstract}
Synthetic turbulence for inflow conditions formulated on a 2-D plane generally produces unphysically large pressure fluctuations in direct numerical and large-eddy simulations. To reduce such artificial fluctuations a divergencefree method is developed with incompressible flow solvers. The procedure of the velocity-pressure solvers is slightly modified on a vertical plane near (rather than at) the inlet by inserting the synthetic turbulence on that plane during the procedure. Simple analytic and numerical error estimations are used to show that the impact of the modified solvers on solution accuracy is small. The final synthetic turbulence satisfies the divergence-free condition. No additional CPU time is required to achieve this condition. The method was tested via simulations of a plane channel flow with $R e_{\tau}=395$. Reynolds stresses, wall skin friction and power spectra of velocity fluctuations are compared with those obtained from using periodic inlet-outlet boundary conditions. In particular, the variances and power spectra of pressure fluctuations are shown to be accurately predicted only when the divergence-free
\end{abstract}

\footnotetext{
*+44 (0)23 80594493

Email address: z.xie@soton.ac.uk (Zheng-Tong Xie )
} 
inlet condition is used.

Keywords: inflow condition, divergence-free, pressure fluctuations, peak loading

\section{Introduction}

Partial differential equations cannot be solved without imposing proper boundary conditions (BCs). In aerodynamics, especially for convective flows, inflow conditions strongly influence the results. Direct Numerical Simulation (DNS) and Large-Eddy Simulation (LES) resolve all the unsteady, threedimensional and energy-containing eddies. For laminar inflow, 'smooth' velocity profiles naturally provide sufficient inlet conditions, whereas for a turbulent inflow appropriate details of the fluctuating motions are required.

Present inflow methods so far fall mainly in two categories. The first is the recycle/rescale method in which inflow data is collected either from a certain point downstream of the same simulation or from an auxiliary simulation. The second is the synthetic approach, in which artificially generated turbulence fluctuations are provided, using random sequences. Usually, statistical information required for representing the inflow turbulence includes first and second moments, space and time correlations and spectra. Comprehensive reviews according to these categories can be found in, for example, Keating et al. [1], Jarrin [2] and Tabor and Baba-Ahmadi [3].

Only a very few papers in literature introduce synthetic inlet turbulence satisfying the divergence-free condition. Smirnov et al. [4] considered the divergence-free condition using a superimposition of harmonic functions to provide synthetic turbulence. Huang et al. [5] improved the Smirnov method 
by imposing von Karman spectra rather than a Gaussian model. Kornev and Hassel [6] derived the velocity potential which satisfies the divergencefree condition and then numerically calculated the solution. Poleto et al. [7] recently proposed a similar method and showed a significant decrease of pressure fluctuations in a turbulent channel flow using their new method.

Nevertheless, none of these authors analysed in any depth the impact of the inflow condition on pressure fluctuations, such as variance and spectra. For many applications the pressure fluctuation field is of primary interest. The major objective in the present work was therefore to develop a more satisfactory method in this regard.

We propose here a divergence-free inflow generation method which is based on Xie and Castro's method [8] (hereafter, XC) with a slight, but crucial, modification of the incompressible flow solvers. This is described in $\S 2$, followed by a simple accuracy analysis. Results of simulations of a plane channel flow and comparisons between these and those obtained using the original method [8] and periodic inlet-outlet boundary conditions, as well as canonical direct numerical simulation (DNS) data for the same flow [10], are presented in $\S 3$. Summary and concluding remarks are followed in $\S 4$.

\section{Methodology}

\subsection{A brief review of the $X C$ [8] inflow condition}

The XC model is a synthetic turbulence generation method and imposes correlations using an exponential function to satisfy the prescribed space and time correlations. The usual relation for the inlet velocities is,

$$
u_{i}=U_{i}+a_{i j} u_{*, j},
$$




$$
\psi_{m}=\sum_{j=-N}^{N} b_{j} r_{m+j},
$$

54 where $N=2 n, n=I / \Delta x, \Delta x$ is grid size and $I$ is an integral length scale. ${ }_{55} \psi$ is the intermediate velocity field and $r$ is a $1 \mathrm{D}$ random number sequence 56 with zero mean and unit variance. $\psi$ is a $1 \mathrm{D}$ number sequence with zero 57 mean, unit variance and spatial correlation. Note that the subscripts, $m, j$, 58 are the position indices. The model constant $b_{j}$ is estimated as

$$
b_{j}=\frac{b_{j}^{\prime}}{\left(\sum_{l=-N}^{N}{b^{\prime}}_{l}^{2}\right)^{1 / 2}} \text { with } b_{j}^{\prime}=\exp \left(-\frac{\pi|j|}{2 n}\right)
$$




$$
u_{*, i}(t+\Delta t)=u_{*, i}(t) \exp \left(-\frac{C_{X C} \Delta t}{T}\right)+\psi_{i}(t)\left[1-\exp \left(-\frac{2 C_{X C} \Delta t}{T}\right)\right]^{0.5},
$$

It is straightforward to generate spatial correlations for a $2 \mathrm{D}$ space (cf. Eq.3) as,

$$
\psi_{m, l}=\sum_{j=-N}^{N} \sum_{k=-N}^{N} b_{j} b_{k} r_{m+j, l+k} .
$$

Only one slice of the 2D signal, $\psi_{m, l}$, is generated at each time and is correlated with the velocity at previous time level using,

where the model constant $C_{X C}=\pi / 4$ and $T$ is the Lagrangian time scale which is estimated using $T=I / U$ where, again, $I$ is a turbulence integral length scale and $U$ is a mean convective velocity. Note that the subscript $i$ is a vector index, i.e. $i=1,2,3$. The process in Eq. 6 effectively imposes an exponential correlation in the streamwise direction. The $\mathrm{XC}$ method generates synthetic turbulence by using Eqs. 1 - 6. In general, the integral length scales, $I$, depend on each velocity component and direction, see Eq. 7.

It is to be noted that in XC the correlation functions were modelled as $C(r)=\exp \left(-\frac{\pi r}{2 I}\right)$. Based on DNS data $[10,11]$ of turbulent channel flows, the exponential model for correlations was examined carefully at different wall-normal distances. If the integral length scale is defined as the enclosed area of the correlation function (Eq. 7), the function $C(r)=\exp \left(-\frac{\pi r}{4 I}\right)$ gives a better fit compared to that used in $\mathrm{XC}$, so is used throughout this paper.

$$
I_{i j}=\int_{0}^{r_{i j, 0.1}} C_{i}\left(r \hat{e}_{j}\right) d r
$$


where $C_{i}\left(r \hat{e}_{j}\right)$ is the correlation function, $i$ and $j$ correspond to the components of the velocity vector and directions, respectively, and $r_{i j, 0.1}$ is the separation distance where $C_{i}\left(r \hat{e}_{j}\right)=0.1$.

\subsection{Inlet mass flux correction}

Ideally the 2D plane of velocity fluctuations generated from Eq. 6 has a zero mean. However usually the mean is not strictly zero because the size of the inlet area is finite in practice. Thus the instantaneous mass flux at the inlet by using the XC model changes very slightly in time. A small fractional difference in the mass flux may lead to significant modifications on the global pressure because of the nature of incompressible flow. Other types of inflow generator might have a similar issue due to the finite number of sampling points or interpolation errors, rather than the specific way of producing the synthetic inflow turbulence. Effects of the non-constant mass flux on the pressure fluctuations were reported in [7] through numerical studies. Artificial pressure fluctuations due to the time dependent mass flux were observed in [12] using a recyling/rescaling inflow method.

We introduce a simple correction to maintain the constant mass flux. Instantaneous velocity at the inlet boundary is corrected as,

$$
\begin{aligned}
& u_{i}=\frac{U_{b}}{U_{b, T}} u_{i, T}, \\
& \text { where, } U_{b, T}=\frac{\int_{S} u_{n, T} d S}{S},
\end{aligned}
$$

where $u_{i, T}$ is the generated velocity from the XC model and $u_{n, T}$ is the component of $u_{i, T}$ normal to the inlet boundary. $S$ is the surface area of the inlet, $U_{b}$ is the prescribed bulk velocity and $U_{b, T}$ is the instantaneous bulk 
velocity calculated from the uncorrected velocities. Simulations which use the corrected velocity in Eq. 8 will be denoted by XCMC. Effects of the mass flux correction on the pressure and velocity fields are reported in $\S 3$.

\subsection{Divergence-free modification}

To satisfy the divergence-free condition, first the generated synthetic turbulence is inserted on a plane near the inlet after having solved the momentum equations. The velocities are then adjusted by the velocity-pressure coupling procedure. This means that, on application of the pressure-correction step, the imposed velocities on the plane where the synthetic turbulence is introduced only act as intermediate velocities. Applying synthetic turbulence on the inlet boundary itself, in contrast, fixes those velocities as final velocities throughout one time step.

Once the synthetic turbulence goes through the velocity-pressure coupling procedure, the velocities are adjusted and are not generally exactly the same as the original. Nevertheless the changes are expected to be small [13]. The important feature of the method presented here is that it does not require any additional computational cost. A brief description of the standard sequence of velocity-pressure coupling procedure with incompressible flow solvers is presented to show the modification for the divergence-free method.

\subsubsection{Velocity and pressure coupling procedure}

The non-dimensionalised incompressible Navier-Stokes equations without any source term, in Cartesian coordinates, are 


$$
\begin{gathered}
\frac{\partial u_{i}}{\partial t}+u_{j} \frac{\partial u_{i}}{\partial x_{j}}=-\frac{\partial p}{\partial x_{i}}+\frac{1}{R e} \frac{\partial \tau_{i j}}{\partial x_{j}} \\
\frac{\partial u_{i}}{\partial x_{i}}=0
\end{gathered}
$$

$$
u_{i, P}^{n+1}=\tilde{u}_{i, P}^{n+1}-\frac{1}{A_{P}}\left(\frac{\partial p^{n+1}}{\partial x_{i}}\right)_{P} .
$$

131 Requiring $u_{i, P}^{n+1}$ to be divergence free and applying the divergence operator 132 on Eq. 14 leads to,

$$
A_{P} u_{i, P}^{n+1}+\sum_{l} A_{l} u_{i, l}^{n+1}=-\left(\frac{\partial p^{n+1}}{\partial x_{i}}\right)_{P}+Q_{i},
$$

$$
u_{i, P}^{n+1}=\frac{Q_{i}-\sum_{l} A_{l} u_{i, l}^{n+1}}{A_{P}}-\frac{1}{A_{P}}\left(\frac{\partial p^{n+1}}{\partial x_{i}}\right)_{P} .
$$

The first term on the right-hand-side (R.H.S.) can be written in a brief form as,

$$
\tilde{u}_{i, P}^{n+1}=\frac{Q_{i}-\sum_{l} A_{l} u_{i, l}^{n+1}}{A_{P}},
$$

Eq. 14 leads

$$
\frac{\partial}{\partial x_{i}}\left[\frac{1}{A_{P}} \frac{\partial p^{n+1}}{\partial x_{i}}\right]_{P}=\left[\frac{\partial \tilde{u}_{i}^{n+1}}{\partial x_{i}}\right]_{P} .
$$


where

$$
u_{i, P}^{* *}=\tilde{u}_{i, P}^{*}+\tilde{u}_{i, P}^{\prime}-\frac{1}{A_{P}}\left(\frac{\partial p^{*}}{\partial x_{i}}\right)_{P}
$$

$$
u_{i, P}^{*}=\tilde{u}_{i, P}^{*}-\frac{1}{A_{P}}\left(\frac{\partial p^{n}}{\partial x_{i}}\right)_{P},
$$

$u_{i}^{*}$ generally does not satisfy the divergence-free condition. To satisfy this requirement, corrections are introduced for both velocity and pressure, $u_{i}^{* *}=$ $u_{i}^{*}+u_{i}^{\prime}, p^{*}=p^{n}+p^{\prime}$. Then the first corrector step is,

${ }_{146} \tilde{u}_{i}^{\prime}$ is neglected at the first corrector step and applying the divergence 147 operator to Eq. 17 to calculate $p^{*}$ yields

$$
\frac{\partial}{\partial x_{i}}\left[\frac{1}{A_{P}} \frac{\partial p^{*}}{\partial x_{i}}\right]_{P}=\left[\frac{\partial \tilde{u}_{i}^{*}}{\partial x_{i}}\right]_{P} .
$$

${ }_{148} \quad$ Note that the corrected velocities $u_{i}^{* *}$ are required to satisfy the divergence149 free condition. The neglected term $\tilde{u}_{i}^{\prime}$ in Eq. 17 can be approximated via 
introducing one further correction, $u_{i}^{* * *}=u_{i}^{* *}+u_{i}^{\prime \prime}, p^{* *}=p^{*}+p^{\prime \prime}$. This leads to the second corrector step,

$$
\begin{aligned}
u_{i, P}^{* * *} & =\tilde{u}_{i, P}^{*}+\tilde{u}_{i, P}^{\prime}-\frac{1}{A_{P}}\left(\frac{\partial p^{* *}}{\partial x_{i}}\right)_{P} \\
& =\tilde{u}_{i, P}^{* *}-\frac{1}{A_{P}}\left(\frac{\partial p^{* *}}{\partial x_{i}}\right)_{P} .
\end{aligned}
$$

The corrected pressure $p^{* *}$ can be calculated requiring that the further corrected velocities $u_{i}^{* * *}$ are divergence free,

$$
\frac{\partial}{\partial x_{i}}\left[\frac{1}{A_{P}} \frac{\partial p^{* *}}{\partial x_{i}}\right]_{P}=\left[\frac{\partial \tilde{u}_{i}^{* *}}{\partial x_{i}}\right]_{P} .
$$

More corrector steps are possible but it has been shown that further corrections are superfluous for most practical purpose [15]. $u_{i}^{* * *}$ and $p^{* *}$ are considered be accurate approximations of the exact solutions, $u_{i}^{n+1}$ and $p^{n+1}$, and they are ready to be used for the next time step. The equations used here are consistent with those in the source code in OpenFOAM v1.7.1 [16] and the literature [e.g. 14] as shown in Appendix A.

\subsubsection{Divergence-free inflow condition method}

Based on the XC method, a new method with divergence-free condition satisfied is suggested and is denoted by XCDF. In Eq. 16, $\tilde{u}_{i}^{*}$ can be considered as the velocity excluding contributions of the pressure gradient [14]. The idea of the divergence-free turbulence method is to let $\tilde{u}_{i}^{*}$ on one $2 \mathrm{D}$ transverse plane near the inlet contain turbulence contents and then correct them with appropriate pressure contributions to satisfy the divergence-free condition. The velocity fluctuations generated from the XC model are imposed appropriately on the $2 \mathrm{D}$ plane at $x=x_{0}$ (see $\S 3$ ), rather than at the 

as,

$$
\begin{gathered}
u_{i, P}^{* *}=\tilde{u}_{i, P}^{g *}-\frac{1}{A_{P}}\left(\frac{\partial p^{*}}{\partial x_{i}}\right)_{P}, \\
\frac{\partial}{\partial x_{i}}\left[\frac{1}{A_{P}} \frac{\partial p^{*}}{\partial x_{i}}\right]_{P}=\left[\frac{\partial \tilde{u}_{i}^{g *}}{\partial x_{i}}\right]_{P},
\end{gathered}
$$

171 where $\tilde{u}_{i}^{g *}$ is defined in the same way as in Eq. 18; Eqs. 17 and 19 are not 172 changed in the rest of the domain. $u_{i}^{g *}\left(x_{0}\right)$ is the generated velocity using

$$
\begin{gathered}
u_{i, P}^{* * *}=\tilde{u}_{i, P}^{g * *}-\frac{1}{A_{P}}\left(\frac{\partial p^{* *}}{\partial x_{i}}\right)_{P}, \\
\frac{\partial}{\partial x_{i}}\left[\frac{1}{A_{P}} \frac{\partial p^{* *}}{\partial x_{i}}\right]_{P}=\left[\frac{\partial \tilde{u}_{i}^{g * *}}{\partial x_{i}}\right]_{P} .
\end{gathered}
$$

183 The same generated velocities as in Eq. 22 are imposed at $x=x_{0}$, i.e. $u_{i}^{g * *}\left(x_{0}\right)=u_{i}^{g *}\left(x_{0}\right)$. Further correction steps are possible but simulations 
showed no further improvement in terms of the development distance of wall skin friction and pressure fluctuations. Thus $u_{i}^{* * *}$ and $p^{* *}$ are considered to be the solution for the next time level. Note that the corrected velocities $u_{i}^{* * *}$, in Eq. 24 are not used to calculate $u_{*, i}(t+\Delta t)$ in Eq. 6 , so that the velocity correction in Eq. 24 would not affect the correlations which are imposed in Eqs. 3 - 6. Further analysis and remarks are presented below in subsections 2.3.3 and 3.3.1.

\subsubsection{Accuracy analysis for the XCDF model}

The PISO algorithm is a non-iterative method in the sense that the momentum equation is solved only once within one time step. Once the velocity is predicted based on the pressure and flux at the previous time level then it is adjusted through several corrector steps. Thus it is important to show that the final corrected velocities are a reasonable approximation. Comprehensive studies by Issa [15] on the accuracy and stability for the PISO algorithm showed that the errors induced in each predictor and corrector step decay with some power of the time step, i.e. $d t^{n}$.

Synthetic turbulence is substituted only on one transverse 2D plane (near the inlet); the velocity-pressure coupling procedure in the rest of the whole domain remains unchanged. We would therefore not expect the the modification to lead to solution divergence. It is nonetheless desirable to consider accuracy and consistency for the sake of reliability of the overall model. We can estimate the decay of errors in terms of $d t$ both analytically and numerically. The analysis presented below, however, should be considered only as a guideline since, like that in [15], it is based on linear partial differential equations. It must be tested in actual computations. Thus the full effects of 
the modification for the XCDF model is analysed and validated in $\S 3$.

Euler time discretization is adopted for the accuracy analysis but other discretization methods should, in principle, provide the same conclusion. As in [15], $A_{P}$ in Eq. 11 is decomposed into two parts, one is for the temporal discretised term and the other is for the rest,

$$
A_{P}=\frac{1}{d t}+A_{P}^{\prime}
$$

For the accuracy analysis, new error terms for velocity and pressure are introduced,

$$
\begin{aligned}
& \varepsilon_{i}^{k}=u_{i}^{n+1}-u_{i}^{k}, \\
& \xi^{l}=p^{n+1}-p^{l},
\end{aligned}
$$

where $k=*, * *, * * *$ and $l=n, *, * *$. Subtracting Eq. 16 from Eq. 14 gives,

$$
A_{P} \varepsilon_{i, P}^{*}=-\sum_{l} A_{l} \varepsilon_{i, l}^{*}-\left(\frac{\partial \xi^{n}}{\partial x_{i}}\right)_{P},
$$

where $\xi^{n}$ is $O(d t)$ via the Taylor series expansion under the Euler discretization scheme, i.e. $\xi^{n}=p^{n+1}-p^{n}=O(d t)$.

Rewriting Eq. 28,

$$
\frac{\varepsilon_{i, P}^{*}}{d t}=-A_{P}^{\prime} \varepsilon_{i, P}^{*}-\sum_{l} A_{l} \varepsilon_{i, l}^{*}-\left(\frac{\partial \xi^{n}}{\partial x_{i}}\right)_{P},
$$

yields $\varepsilon_{i}^{*}=O\left(d t^{2}\right)$.

In a similar way, we subtract Eqs. 22, 23 from Eqs. 14, 15 and get the error equations on the 2D plane where synthetic turbulence is imposed,

$$
A_{P} \varepsilon_{i, P}^{* *}=-\sum_{l} A_{l} \varepsilon_{i, l}^{g *}-\left(\frac{\partial \xi^{*}}{\partial x_{i}}\right)_{P},
$$


and

$$
\frac{\partial}{\partial x_{i}}\left[\frac{1}{A_{P}} \frac{\partial \xi^{*}}{\partial x_{i}}\right]_{P}=\frac{\partial}{\partial x_{i}}\left[-\frac{1}{A_{P}}\left(\sum_{l} A_{l} \varepsilon_{i, l}^{g *}\right)\right]_{P},
$$

where $\varepsilon_{i}^{g *}=u_{i}^{n+1}-u_{i}^{g *}$. It is difficult to accurately estimate $\varepsilon_{i}^{g *}$ at this stage. Nevertheless it is inherently no greater than the full difference of the generated (uncorrected) velocities between the time steps $n+1$ and $n$. When the time indices are $t+\Delta t \rightarrow n+1$ and $t \rightarrow n$ in Eq. 6 , the full difference of the generated velocity, $\varepsilon_{i}^{g}$, can be estimated by combining Eqs. 1 and 6 ,

$$
\begin{aligned}
\varepsilon_{i}^{g} & =a_{i j}\left(u_{*, i}^{n+1}-u_{*, i}^{n}\right) \\
& =a_{i j}[-u_{*, i}^{n} \underbrace{\left(1-e^{\left(-\frac{C_{X T}}{T} d t\right)}\right)}_{\sim O(d t)}+\psi_{i}^{n} \underbrace{\left(1-e^{\left(-2 \frac{C_{X T}}{T} d t\right)}\right)^{0.5}}_{\sim O(d t)}] .
\end{aligned}
$$

Then it is estimated that $\varepsilon_{i}^{g}=O(d t)$ and $\varepsilon_{i}^{g *}=O(d t)$. We assume $\varepsilon_{i}^{*}=O\left(d t^{2}\right)$ is still valid for the pressure in the rest of the domain (i.e. except for $x=x_{0}$ ). Then the velocity error along the streamwise direction, i.e. $\varepsilon_{i}^{*}=O\left(d t^{2}\right)$ (for $\left.x \neq x_{0}\right)$ and $\varepsilon_{i}^{g *}$ (for $x=x_{0}$ ) is in a Dirac delta function form. Note in Eq. 31, the L.H.S. term is a second order spatial derivative, whereas the R.H.S. term is a first order spatial derivative. As usual for simpler analyses, we start by considering a 1D form of Eq. 31 in which synthetic velocity fluctuations are imposed only at $x=x_{0}$. Given that non-dimensional equations are being used and the CFL number $d t \times u / d x \sim 1$, integrating Eq. 31 in space leads to $\xi^{*}=O\left(d x \varepsilon^{g *}\right)=O\left(d t \varepsilon^{g *}\right)$. Nevertheless, our real problem is $3 \mathrm{D}$ and thus it is difficult to give an accurate estimation for $\xi^{*}$ in terms of $\varepsilon^{g *}$. If we 
agree that neither $\xi^{*}=O\left(\varepsilon_{i}^{g *}\right)$ nor $\xi^{*}=O\left(d t \varepsilon_{i}^{g *}\right)$ is an accurate estimation, perhaps $\xi^{*}=O\left(d t^{\beta} \varepsilon_{i}^{g *}\right)=O\left(d t^{1+\beta}\right)$ is a slightly better one, where $0<\beta<1$.

Using Eq. 30 and following the same procedure as for the estimation for $\varepsilon_{i}^{*}$, we obtain $\varepsilon_{i}^{* *}=O\left(d t^{2}\right)$. It is to be noted that $\xi^{*}=O\left(d t^{1+\beta}\right)$ and $\varepsilon_{i}^{* *}=$ $O\left(d t^{2}\right)$ are the worst errors at $x=x_{0}$; further downstream the errors reduce to the levels suggested in [15].

The errors in the second corrector step are calculated by subtracting Eqs. 24 and 25 from Eqs. 14 and 15. The magnitudes of the errors from this step are the same as those in the first corrector step because the same generated velocity is imposed at the second corrector too. Thus the maximum errors are $\xi^{* *}=O\left(d t^{1+\beta}\right)$ and $\varepsilon_{i}^{* * *}=O\left(d t^{2}\right)$ on the transverse plane $x=x_{0}$.

This analysis has revealed that the maximum velocity error (i.e. at $x=$ $\left.x_{0}\right)$ is one order higher than the truncation error (i.e. $\sim O(d t)$ for the Euler discretisation). However, the maximum pressure error is less than one order higher than the truncation error $O(d t)$. As discussed earlier, the modification is applied only on one 2D plane. Again it is expected that the errors are not significant near the plane and decay downstream to the levels suggested in $[15]$.

In order to get more confidence in the error analysis, the decay of the errors are numerically calculated for plane channel flows and are compared with those using periodic in-outlet boundary conditions (PBC). The computational details for the two cases are identical except for the inflow conditions. Details of the numerical settings are presented in $\S 3$. The spanwise- and timeaveraged profiles of the errors for velocity and pressure are presented in Fig.1. Note that the velocity error from case XCDF is based on the generated ve- 
locity field on the plane at $x=x_{0}$, i.e. $\left|\varepsilon^{*}\right|=\left|u_{1}^{n+1}-u_{1}^{g *}\right|$, and the pressure error $\xi^{*}$ is that defined in Eqs. 27 and 31 . Note we are not able to get exact solutions $u_{i}^{n+1}$ and $p^{n+1}$ as in Eq.27, thus the final numerical solutions are used instead.

It is not surprising that the absolute magnitudes of the velocity errors for case XCDF are significantly greater than those for case PBC in Fig. 1(a). However, the error decay with time step for case XCDF is similar to that for case PBC shown in the inset at the upper corner of the figure. Both cases clearly show that as $d t \rightarrow 0$, the velocity errors decay towards zero at a rate close to $d t^{-1}$, confirming the $\varepsilon_{i}^{g *}=O(d t)$ behaviour estimated analytically. Again, the errors at $x=x_{0}$ shown here are the worst for case XCDF, whereas in the regions downstream (i.e. $x / \delta>5$ ), they are close to those for case PBC. This confirms that the errors decays downstream to the levels suggested in $[15]$.

The pressure errors for cases $\mathrm{PBC}$ and $\mathrm{XCDF}$ in Fig. 1(b) show rather similar magnitude as that of the velocity errors. However the decay rate of the pressure errors for case XCDF seems significantly slower than that of the velocity errors. This is because the pressure errors are also affected by the spatial discretization error as in Eq. 31. In these tests the mesh size was fixed in order to check the error decay rates in terms of time step, and also to save computational cost. It is expected that varying the grid size with the time step and keeping the CFL number $d t \times u / d x$ unchanged would lead to a faster decay rate of the pressure errors for case XCDF. Because it is impossible to get the exact solutions, the numerical procedure for the error estimation is not identical to the analytic procedure discussed 
earlier. Nevertheless, the former in general confirms those suggested by the latter. Again the numerical procedure shows that the errors in velocities and pressure decrease with decreasing time step $d t$. This suggests that our modification with the PISO procedure is self-consistent.

\section{Validations of turbulent inflow conditions on a plane channel flow}

The XC, XCMC and XCDF methods are used as inflow conditions to simulate a plane channel flow. These models are assessed through a validation against using periodic in-outlet boundary conditions (PBC) for the plane channel flow. The purpose of using periodic simulation data (as done in a number of previous papers - e.g. $[1,8,18,19,20,21])$ is simply to provide a straightforward validation for the inflow method without the other uncertainties which would inevitably arise when using non-periodic test cases. Once the method is validated on a channel flow, it can be used for both free and wall-bounded flows. The input parameters, such as Reynolds stresses and integral length scales, can be obtained from the available experimental data and/or appropriate empirical stress ratios [e.g. 22].

\subsection{Numerical description}

The Reynolds number of the channel flow based on the friction velocity, $u_{\tau}$ and the half depth of the channel, $\delta$, was $R e_{\tau}=395$. The domain size was $60 \delta \times 2 \delta \times 3.5 \delta$ in the streamwise $(x)$, wall-normal $(y)$ and spanwise (z) directions respectively (see Fig. 2). A uniform mesh was used in the streamwise and spanwise directions and a stretched mesh in the wall-normal 
direction for which $y_{1}^{+} \leq 1$ was satisfied at the first cell centre. The number of grid points was $600 \times 60 \times 70$ in the $x, y$ and $z$ directions respectively. The resolutions in the $x$ and $z$ directions were $\Delta x^{+}=39.5$ and $\Delta z^{+}=19.8$.

All statistics were averaged over $40 t_{*}$, where $t_{*}=t u_{\tau} / \delta$, and the averaging started after an initialization period of $20 t_{*}$. The Smagorinsky subgrid-scale model with van-Driest damping [23] was adopted with the constant $C_{s}=$ $0.065[24]$. The time step satisfied the condition that the CFL number was less than unity, corresponding to $\Delta t_{*}=\Delta t \times u_{\tau} / \delta=0.002$. A second order, implicit scheme was used for time discretization, with a second order central difference scheme for spatial discretization. The transient incompressible flow solver in OpenFOAM 1.7.1 [16] was used and the PISO algorithm was adopted for the velocity-pressure coupling for most of the simulations. The number of pressure correctors was set to two. We noticed that increasing the number of correctors did not improve the results.

A periodic boundary condition was applied in the spanwise direction and no-slip wall boundary conditions were applied on the bottom and top walls for all cases. Other boundary conditions are summarized in Table 1. For the $\mathrm{XCDF}$ model, generated synthetic turbulence from Eq. 1 by using the XC model was imposed at the cell centres of a $y z$ plane which was placed in the domain near the domain inlet, e.g. at $x=x_{0}=\delta$ rather than at the inlet boundary (i.e. $x=0$ ). Meanwhile, the mean velocity profile was specified at the domain inlet to fix the mass flow rate to a constant. Ideally, the shifted inflow plane is to be placed as close as possible to the inlet boundary to save the computational cost. The XCDF model work well for $x_{0} \geq 0.5 \delta$. However, we noticed that placing the plane at the centres of the first cell from the inlet, 
generates higher peaks of time- and spanwise- averaged variance of the wall pressure fluctuations near the inlet. This might be due to the fixed mean velocity specified at the inlet boundary and the nature of the incompressible flow. Nevertheless, the magnitude of these pressure variance peaks are far less than those generated by the XCMC model.

\subsection{Specifying input parameters}

The XC, XCMC and XCDF models need first and second moment statistics and integral length scales as input parameters. These were taken from DNS data [10] and case PBC. The mean velocity and Reynolds stress profiles for case PBC are compared with reference DNS data in Fig. 3. Overpredictions of mean velocity at the channel centre and $\left\langle u^{\prime} u^{\prime}\right\rangle^{+}$near the wall, and under-predictions of $\left\langle v^{\prime} v^{\prime}\right\rangle^{+}$near the wall are common observations in LES with a similar resolution [e.g. 2, 25].

The integral length scales were calculated by integrating two-point correlations until the value of the correlation reached 0.1 . The correlations are taken from DNS data [10]. Nine integral length scales were estimated for the three components of the velocity vector $(u, v, w)$ in all three directions $(x, y, z)$ (see Eq. 7). For instance, the integral length scale in the spanwise direction $(j=3)$ for the correlation $C_{i}(i=1)$ (i.e. based on the $u_{1}$ component) is $I_{13}$. The channel flow in the wall-normal direction is inhomogeneous thus $I_{i 2}$ cannot be obtained by using Eq. 7. For simplicity, it was assumed that $I_{i 2}=I_{i 3}$. Fig. 4 shows the integral length scales used for the input data of the XC, XCMC and XCDF models. It is to be noted that we managed to use as much as possible the available reference data to have a rigorous test. In more practical applications, it is straightforward to use fewer integral length 
scales.

For the inflow models, the distribution of the $x$-direction length scales, $I_{i 1}$, along the wall-normal direction is a function of the local mean velocity, $U_{1}(y)$. Only one 2-D slice of the signal is generated and convected into the domain at every time step. Thus we get $I_{i 1}(y)=T_{i 1} \times U_{1}(y)$ using Taylor's hypothesis where $T_{i 1}$ is the Lagrangian time scale. The local turbulence intensity is mostly far less than 0.3 for the test case, thus Taylor's hypothesis holds across the domain [26]. Implementations of the generated velocity by the models were performed on a virtual uniform mesh and then they were interpolated to the non-uniform mesh at the inlet.

\subsection{Results and discussion}

In the XCDF model, the synthetic turbulence is imposed on a transverse plane at $x=x_{0}$ and is adjusted through the velocity-pressure coupling procedure. The changes are expected to be small, otherwise the Reynolds stresses and the integral length scales used to generate the synthetic turbulence must be reconsidered. Fig. 5 shows a typical example of the changes of time series of the streamwise velocity before and after the continuity equation is satisfied. As expected, the difference between the two sets of velocities is very small.

An accurate prediction of the pressure fluctuations is the focus of the present paper. Fig. 6(a) show the effects of the mass flux correction and the divergence-free modification on the dimensionless time- and spanwiseaveraged variance of the normalised wall pressure fluctuations, $<p_{w}^{\prime 2}>^{+}=<$ $p_{w}^{\prime 2}>/\left(\rho^{2} u_{\tau}^{4}\right)$. Significantly high wall pressure fluctuations are introduced by the $\mathrm{XC}$ model near the inlet, and they decrease monotonically to zero 
at the outlet where the pressure was fixed to a constant ambient pressure. In contrast, the variances of the wall pressure fluctuations for both cases $\mathrm{XCMC}$ and $\mathrm{XCDF}$ are in good agreement with the reference data (i.e. PBC) downstream from $x / \delta=10$. The simple mass correction in case XCMC brings a significant improvement on pressure fluctuations and its performance in Fig. 6(a) seems similar to that of case XCDF. However, the generated inflow synthetic turbulence in case XCMC does not satisfy the divergencefree condition, and there must be some signature for this.

In checking the Probability Density Functions (PDFs) of the pressure fluctuations sampled at various stations at the centre of the channel (see Fig. 7), we observed more extreme peak pressure fluctuations in case XCMC compared to case XCDF. Fig. 7 shows that the occurrence of extreme peak pressure fluctuations for case XCMC can be more than twice that for case XCDF. This certainly shows an good feature of the XCDF model.

Unphysical peaks near the inlet are generated for both cases where the synthetic turbulence was imposed. Case XCMC gave an order higher pressure fluctuations near the inlet compared with case XCDF (see the inset in Fig. 6(a)). The XCMC model may thus be less satisfactory than the XCDF model if the region of interest is close to the inlet.

Fig. 6(b) show profiles of the dimensionless time- and spanwise-averaged variance of the pressure fluctuations, $<p^{2}>^{+}=<p^{2}>/\left(\rho^{2} u_{\tau}^{4}\right)$, at different downstream locations. The pressure fluctuations for case XCDF downstream from $x / \delta=10$ and for case XCMC from $x / \delta=20$ are in an excellent agreement with the reference data (i.e. PBC). Note that the $\left\langle p^{2}\right\rangle^{+}$for case $\mathrm{XC}$ is far too large to be shown in Fig. 6(b). 
It is of interest to check the turbulence statistics profiles. As a typical example, Fig. 8 present the time- and spanwise-averaged velocity and velocity fluctuation variances at $x=20 \delta$ obtained from using different inflow methods. All of the quantities are normalised appropriately by friction velocity $u_{\tau}$ and they all show a good performance when compared with the reference - case PBC. This suggests that the three inflow models are in a similar performance in this aspect.

The flow development in terms of the recovery distances of wall shear stress and Reynolds shear stress in crucial for the inflow methods. Fig. 9 (a) shows dimensionless wall shear stress $\tau_{w}^{+}=\tau_{w} /\left(\rho u_{\tau}^{2}\right)$. In spite of the significantly different pressure fluctuations between cases XC and XCMC shown in Fig. 6, the wall shear stress and Reynolds shear stress profiles for both cases are almost identical as shown in Fig. 9. The development distance in terms of the recovery of the wall shear stress and Reynolds shear stress for the $\mathrm{XC}$ and $\mathrm{XCMC}$ models is $x / \delta \approx 10$, which is similar to those in Deck et al. [27] in which a turbulent boundary layer was simulated using a different synthetic turbulence inflow method [2]. The development distance for case $\mathrm{XCDF}$ is noticeably greater than those for cases $\mathrm{XC}$ and XCMC, partly because the effective inflow plane for case XCDF is at $x_{0}=\delta$. Nevertheless, the error of wall shear stress at $x / \delta=15$ for case XCDF is within $5 \%$. Setting the $5 \%$ error as the criteria to define the development distance, then it is $14 \delta$ for the XCDF model counting from the plane at $x_{0}$.

Fig. 9(b) shows dimensionless Reynolds shear stress profiles $-\left\langle u^{\prime} v^{\prime}\right\rangle^{+}=$ $-\left\langle u^{\prime} v^{\prime}\right\rangle / u_{\tau}^{2}$. The error of Reynolds shear stress at $(x / \delta=10, y / \delta=0.1)$ for case XCDF is about within $5 \%$. 
To visualize the near wall structures, the second invariant of the velocity gradient tensor can be used - often called the $Q$-criterion (e.g. [28]), which is written as,

$$
Q=\frac{1}{2}\left(\Omega_{i j} \Omega_{i j}-S_{i j} S_{i j}\right),
$$

where $\Omega_{i j}=\left(\frac{\partial u_{i}}{\partial x_{j}}-\frac{\partial u_{j}}{\partial x_{i}}\right) / 2$ and $S_{i j}=\left(\frac{\partial u_{i}}{\partial x_{j}}+\frac{\partial u_{j}}{\partial x_{i}}\right) / 2$. Essentially $Q$ is the balance between the rotation $\left(\Omega_{i j}\right)$ and strain $\left(S_{i j}\right)$ rates. Thus a positive value of $Q$ indicates that the strength of rotation overcomes that of the strain. Fig. 10 shows the iso-surface $Q=200$ in the upstream region of the domain for XCMC and XCDF models. XCMC shows a delay of development of near-wall structures, which is consistent with Fig.9(a). However, XCMC and XCDF models show almost same performance downstream of $x / \delta=10$.

Power Spectral Densities (PSD) for the pressure fluctuations and streamwise velocity fluctuations at two typical stations are shown in Fig. 11. These are consistent with Figs. 6 and 9. The PSD of the pressure fluctuations for case XC (in which the constant mass flux condition is not satisfied) is overpredicted by orders of magnitude through much of frequency range, whereas those for cases XCMC and XCDF show a reasonable agreement with the reference data $(\mathrm{PBC})$ at $x / \delta=10$ and even better agreement at $x / \delta=55$. Spectra of the streamwise velocity fluctuations for all cases are in good agreement at most frequencies in Figs. 11(c) and 11(d). Case XC over-predicts the velocity spectra only at high frequencies.

\subsubsection{Remarks on the XCDF model}

It is a significant challenge to solve the divergence-free problem which arises in applying synthetic inflow conditions, especially since the latter should include crucial features like turbulence integral length scales, spectra, 
Reynolds stresses, anisotropy and inhomogeneity, and whilst maintaining high computational efficiency. Our proposed divergence-free XCDF model certainly is not free of limitations. The development distance in terms of the skin friction needs to be improved if estimation of the skin friction is of major interest. Combining the XCDF model with some up-to-date stochastic forcing methods such as [29] would improve the development distance. Imposing the synthetic turbulence on a transverse plane near the domain inlet does increase computational resources by less than $2 \%$ for these test cases, hence this overhead is negligible.

Based on the statistics from the current test cases, the divergence-free inflow method does not seem to be superior in all aspects compared to a simple mass flux correction approach. However, XCDF has distinctive features. Firstly, the mass correction factor, $U_{b} / U_{b, T}$, in Eq. 8 ranges $1 \pm 0.02$ for the current test case which is relatively high considering $U_{b} / u_{\tau}=18.33$. In practical applications, a very coarse mesh at the inlet may be adopted (i.e. fewer sampling points). And subsequently the mass correction factor can be even greater which can lead to a noticeable alteration in prescribed Reynolds stress in Eq. 2. In such situations, one could argue that the mass flux correction effectively modifies the input turbulence parameters. Secondly, there is an unphysical peak of pressure fluctuations near the inlet as shown in Fig. 6(a). It decays rapidly but may cause unphysical and unacceptably high noise levels for some aeroacoustic applications, especially when the region of interest is inevitably close to the inlet. Thirdly, we noticed that the XCMC model generated more extreme peak pressure fluctuations at the middle of channel compared to case XCDF, though these are not clearly shown in the 
spectra of pressure fluctuations.

The modification to the PISO algorithm is similar in some respects to the body-force approach, e.g. [30, 31, 29]. However, there are clear differences too. For example, in [31], the stochastic force is isotropic. XCDF, on the other hand, can reproduce specified anisotropy by providing individual Reynolds stresses and integral length scales. Also no empirical constant is involved in the XCDF model, unlike in typical body-force approaches.

Laraufie et al. [29] suggests that the development distance decreases with increasing Reynolds number thus applicability of the XCDF model will likely improve further for spatially developing flows at high Reynolds numbers. For example, we have used the XCDF model to simulate surface pressure fluctuations on the Commonwealth Advisory Aeronautical Council (CAARC) standard building at a Reynolds number $3 \times 10^{5}$ based on the free stream velocity and the height of the building [32]. The validation against wind tunnel experiments has been very promising.

The divergence-free model can be easily implemented in other CFD codes. For example, a similar method has been used in an in-house code [33]. Our method has been tested using both PISO and PIMPLE (i.e a combination of PISO and SIMPLE) solvers in OpenFOAM, which suggests the significant potential of the method.

\section{Conclusions}

A new divergence-free synthetic turbulence inflow technique has been developed with incompressible flow solvers. To satisfy the divergence-free criterion, the velocity-pressure coupling (PISO) procedure is modified slightly 
by substituting the generated synthetic turbulence for the intermediate velocities on a transverse plane near the domain inlet before the corrector steps are performed. The synthetic turbulence is mildly adjusted through the correctors and thus is divergence-free. It is to be stressed that this modification of the PISO procedure costs no additional CPU time.

The effects of the modification of the PISO algorithm on solution accuracy have been examined analytically and numerically. The maximum error (always on the transverse plane where synthetic turbulence is imposed) is, for the velocity, one order higher than the truncation error, whereas the maximum error for the pressure is less than one order higher than the truncation error. This is not surprising because imposing of the synthetic turbulence within the domain (rather than at the inlet) is similar in some respects to a body-force approach. Maximum disturbances occur where the synthetic turbulence is imposed. Nevertheless, the errors decay downstream (e.g. $x / \delta>5$ ) to the levels suggested in [15].

The suggested divergence-free turbulence inflow model XCDF has been tested on a channel flow and compared with the XC model [8] and the XC model with a mass flux correction - XCMC. Both XCDF and XCMC give very significant improvements on the computed pressure fluctuations. For example, the variance and spectra of the pressure fluctuations are in good agreement with reference data obtained from a plane channel flow using axially periodic boundary conditions. In addition, the XCDF model is genuinely divergence-free and provides solution improvements in other respects too, such as more reasonable peak pressure fluctuations.

In applications where only time averaged pressure and aerodynamic forces 
(e.g. mean lift and drag on a wind turbine blade or mean wind loads on a building) are of interest, the XC and XCMC models are generally satisfactory. However, if instantaneous forces (e.g. peak structural wind loads in wind engineering applications) are the focus, the divergence-free method XCDF is recommended. In particular, the XCDF method can be very useful in some applications in which the turbulence motions are required to insert in the computation domain. For example, the XCDF method can be used at the interfaces of the coupling of a weather-scale model and a street-scale LES model to provide sufficient turbulent fluctuations when nested meshes are used.

\section{Acknowledgements}

YK acknowledges provision of a Ph.D studentship from the Faculty of Engineering and the Environment, University of Southampton. All the computations were performed on IRIDIS3 at the University of Southampton.

\section{References}

[1] Keating A, Piomelli U, Balaras E, Kaltenbach HJ . a priori and a posteriori tests of inflow conditions for large-eddy simulation. Phys Fluids 2004;16:4696-712.

[2] Jarrin N . Synthetic inflow boundary conditions for the numerical simulation of turbulence. Ph.D. thesis; University of Manchester; 2008. 
[3] Tabor GR, Baba-Ahmadi MH . Inlet conditions for large eddy simulation: A review. Comput Fluids 2010;39:553-67.

[4] Smirnov A, Shi S, Celik I . Random flow generation technique for large eddy simulations and particle-dynamics modeling. J Fluids Eng 2001;123:359-71.

[5] Huang SH, Li QS, Wu JR . A general inflow turbulence generator for large eddy simulation. J Wind Eng Ind Aerodyn 2010;98:600-17.

[6] Kornev N, Hassel E . Synthesis of homogeneous anisotropic divergencefree turbulent fields with prescribed second-order statistics by vortex dipoles. Phys Fluids 2007;19.

[7] Poletto R, Revell A, Craft T, Jarrin N . Divergence free synthetic eddy method for embedded LES inflow boundary conditions. Seventh Int Symp Turb Shear Flow Phenom (Ottawa) 2011;

[8] Xie ZT, Castro IP . Efficient generation of inflow conditions for large eddy simulation of street-scale flow. Flow Turb Combust 2008;81:44970.

[9] Lund TS, Wu X, Squires KD . Generation of turbulent inflow data for spatially developing boundary layer simulations. J Comput phys 1998;140:233-58.

[10] Moser RD, Kim J, Mansour NN . Direct numerical simulation of turbulent channel flow up to $\operatorname{Re}_{\tau}=590$. Phys Fluids 1999;11:943-5. 
[11] Iwamoto K . Databased for fully developed channel flow. Tech. Rep.; Dept. Mech. Eng., Univ. Tokyo; 2002. URL http://www.thtlab.t.u-tokyo.ac.jp/.

[12] Gungor AG, Sillero JA, Jiménez J . Pressure statistics from direct simulation of turbulent boundary layer. Seventh Int Conf Compt Fluid Dyn (Hawaii) 2012;

[13] Kondo K, Murakami S, Mochida A . Generation for velocity fluctuations for inflow boundary condition of LES. J Wind Eng Ind Aerodyn 1997;67:51-64.

[14] Ferziger JH, Perić M . Computational Methods for Fluid Dynamics. Springer; 2002, p. 157-216.

[15] Issa RI . Solution of the implicitly discretised fluid flow equations by operator-splitting. J Compt Phys 1985;62:40-65.

[16] OpenFOAM . User guide. Tech. Rep.; OpenFOAM®; 2010.

[17] Counihan J . An improved method of simulating an atmospheric boundary layer in a wind tunnel. Atmos Environ 1969;3:197-214.

[18] Jarrin N, Benhamadouche S, Laurence D, Prosser R . A synthetic-eddymethod for generating inflow conditions for large-eddy simulations. Int J Heat and Fluid Flow 2006;27:585-93.

[19] Batten P, Goldberg U, Chakravarthy S . Interfacing statistical turbulence closures with large-eddy simulation. AIAA J 2004;42:485-92. 
[20] Klein M, Sadiki A, Janicka J . A digital filter based generation of inflow data for spatially developing direct numerical or large eddy simulations. J Compt Phys 2003;186:652-65.

[21] Veloudis I, Yang Z, McGuirk JJ, Page GJ, Spencer A . Novel implementation and assessment of a digital filter based approach for the generation of LES inlet conditions. Flow Turb Combust 2007;79:1-24.

[22] Xie ZT, Castro IP . Large-eddy simulation for flow and dispersion in urban streets. Atmos Environ 2009;43:2174-85.

[23] van Driest ER . On turbulent flow near a wall. AIAA 1956;23:1007-11.

[24] Moin P, Kim J . Numerical investigation of turbulent channel flow. J Fluid Mech 1982;118:341-77.

[25] Boppana VBL, Xie ZT, Castro IP . Large-Eddy Simulation of dispersion from line sources in a turbulent channel flow. Flow Turb Combust 2012;88:311-42.

[26] Willis GE, Deardorff JW . On the use of Taylor's translation hypothesis for diffusion in the mixed layer. Quart J Roy Meteor Soc 1976;102:81722.

[27] Deck S, Weiss PÉ, Pamiès M, Garnier E . Zonal detached eddy simulation a spatially developing flat plate turbulent boundary layer. Compt Fluids 2011;48:1-15.

[28] Dubief Y, Delcayre F . On coherent-vortex identification in turbulence. J Turb 2000;1:1-22. 
[29] Laraufie R, Deck S, Sagaut P . A dynamic forcing method for unsteady turbulent inflow conditions. J Comput Phys 2011;230:8647-63.

[30] Keating A, Piomelli U . A dynamic stochastic forcing method as a wall-layer model for large-eddy simulation. J Turbul 2006;7,N12.

[31] Keating A, Prisco GD, Piomelli U . Interface conditions for hybrid RANS/LES calculations. Int J Heat Fluid Flow 2006;27:777-88.

[32] Daniels SJ, Castro IP, Xie ZT . Peak loading and surface pressure fluctuations of a tall model building. J Wind Eng Ind Aerodyn 2013; under review.

[33] Piomelli U, Balaras E, Pasinato H, Squires KD, Spalart PR . The innerouter layer interface in large-eddy simulations with wall-layer models. Int J Heat Fluid Flow 2003;24:538-50. 
and

$$
\frac{\partial}{\partial x_{i}}\left[\frac{1}{A_{P}} \frac{\partial}{\partial x_{i}}\left(p^{n}+p^{\prime}\right)\right]_{P}=\left[\frac{\partial}{\partial x_{i}}\left(\tilde{u}_{i}^{*}+\tilde{u}_{i}^{\prime}\right)\right]_{P}-\left[\frac{\partial u_{i}^{* *}}{\partial x_{i}}\right]_{P},
$$

Notations for the PISO algorithm in literature and some source codes may be confusing for whom is not fully aware of the method. Therefore consistency among the equations used in this study, OpenFOAM code v1.7.1 (OF) $[16]$ and Ferziger and Perić [14] are shown here for reader's convenience. Taking the divergence, Eqs. 17 and 16 are rewritten respectively as,

$$
\frac{\partial}{\partial x_{i}}\left(\frac{1}{A_{P}} \frac{\partial p^{n}}{\partial x_{i}}\right)_{P}=\left[\frac{\partial \tilde{u}_{i}^{*}}{\partial x_{i}}\right]_{P}-\left[\frac{\partial u_{i, P}^{*}}{\partial x_{i}}\right]_{P} .
$$

$$
\frac{\partial}{\partial x_{i}}\left(\frac{1}{A_{P}} \frac{\partial p^{\prime}}{\partial x_{i}}\right)_{P}=\left[\frac{\partial u_{i}^{*}}{\partial x_{i}}\right]_{P} .
$$

This equation is identical to Eq. 7.39 in [14].

Neglecting $\tilde{u}_{i}^{\prime}$ in Eq. 17 and subtracting the equation from Eq. 20 leads,

$$
u_{i, P}^{\prime \prime}=\tilde{u}_{i, P}^{\prime}-\frac{1}{A_{P}}\left(\frac{\partial p^{\prime \prime}}{\partial x_{i}}\right)_{P},
$$

which is identical to Eq. 7.43 in [14]. Taking divergence and requiring $\partial u_{i}^{\prime \prime} / \partial x_{i}=0$ (note $u_{i}^{* *}$ and $u_{i}^{* * *}$ are divergence free), Eq. A.4 is written as,

$$
\frac{\partial}{\partial x_{i}}\left[\frac{1}{A_{P}} \frac{\partial p^{\prime \prime}}{\partial x_{i}}\right]_{P}=\left[\frac{\partial \tilde{u}_{i}^{\prime}}{\partial x_{i}}\right]_{P},
$$

This is identical as Eq. 7.44 in [14]. 


\section{Appendix C. pisoFOAM.C in OpenFOAM v.1.7.1}

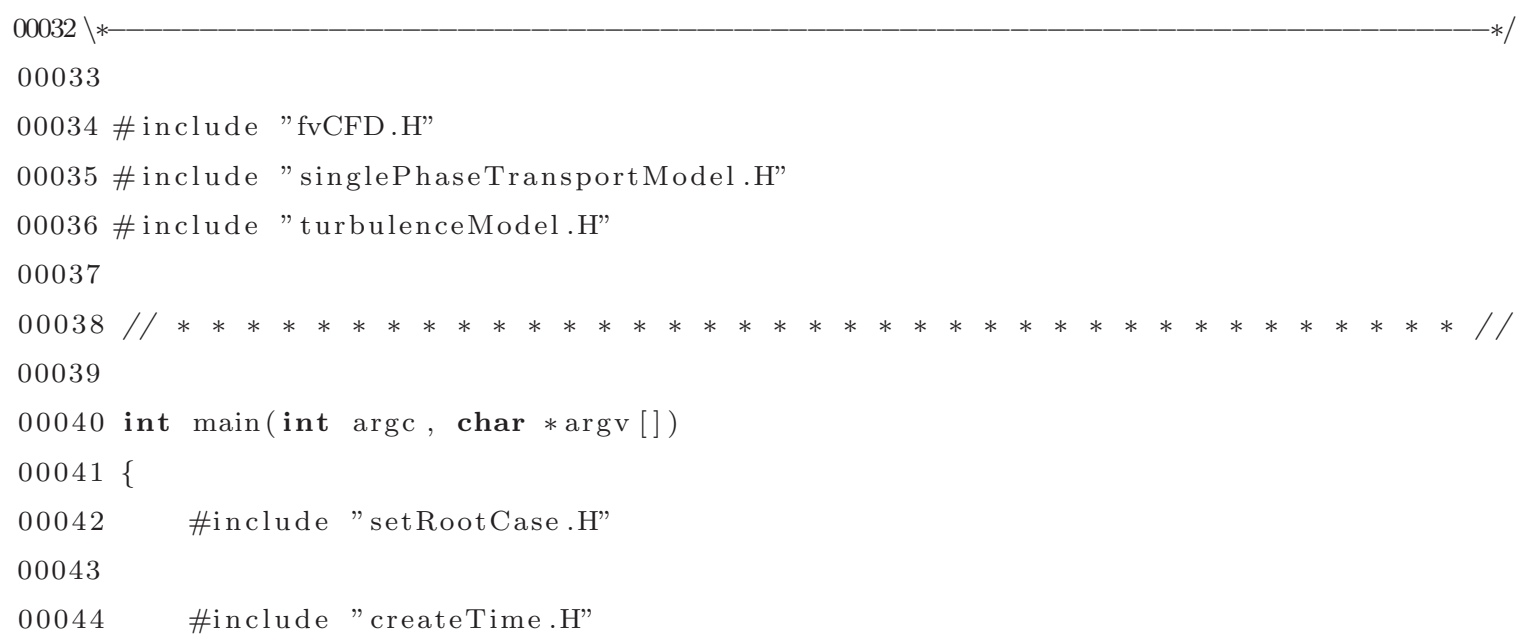




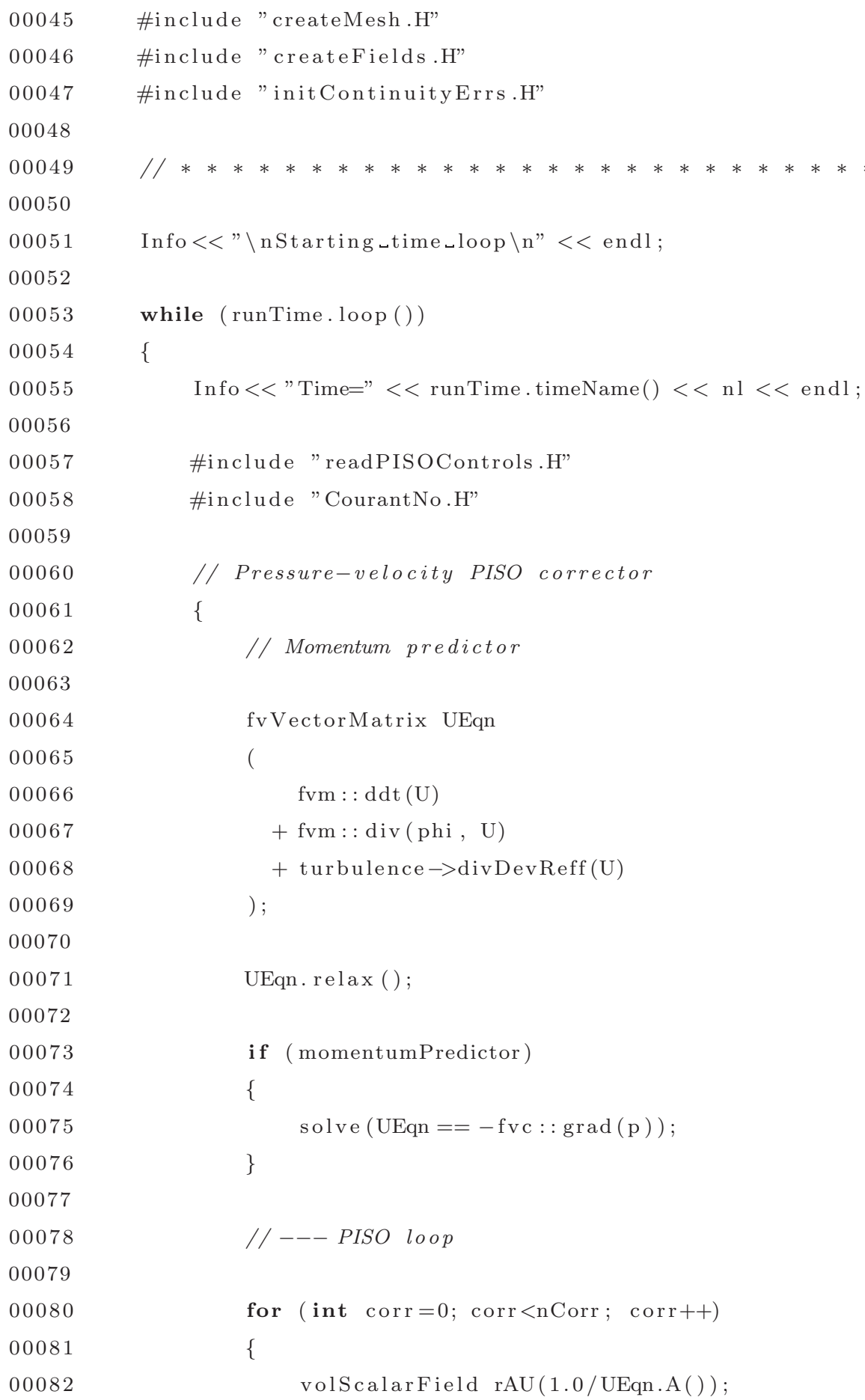




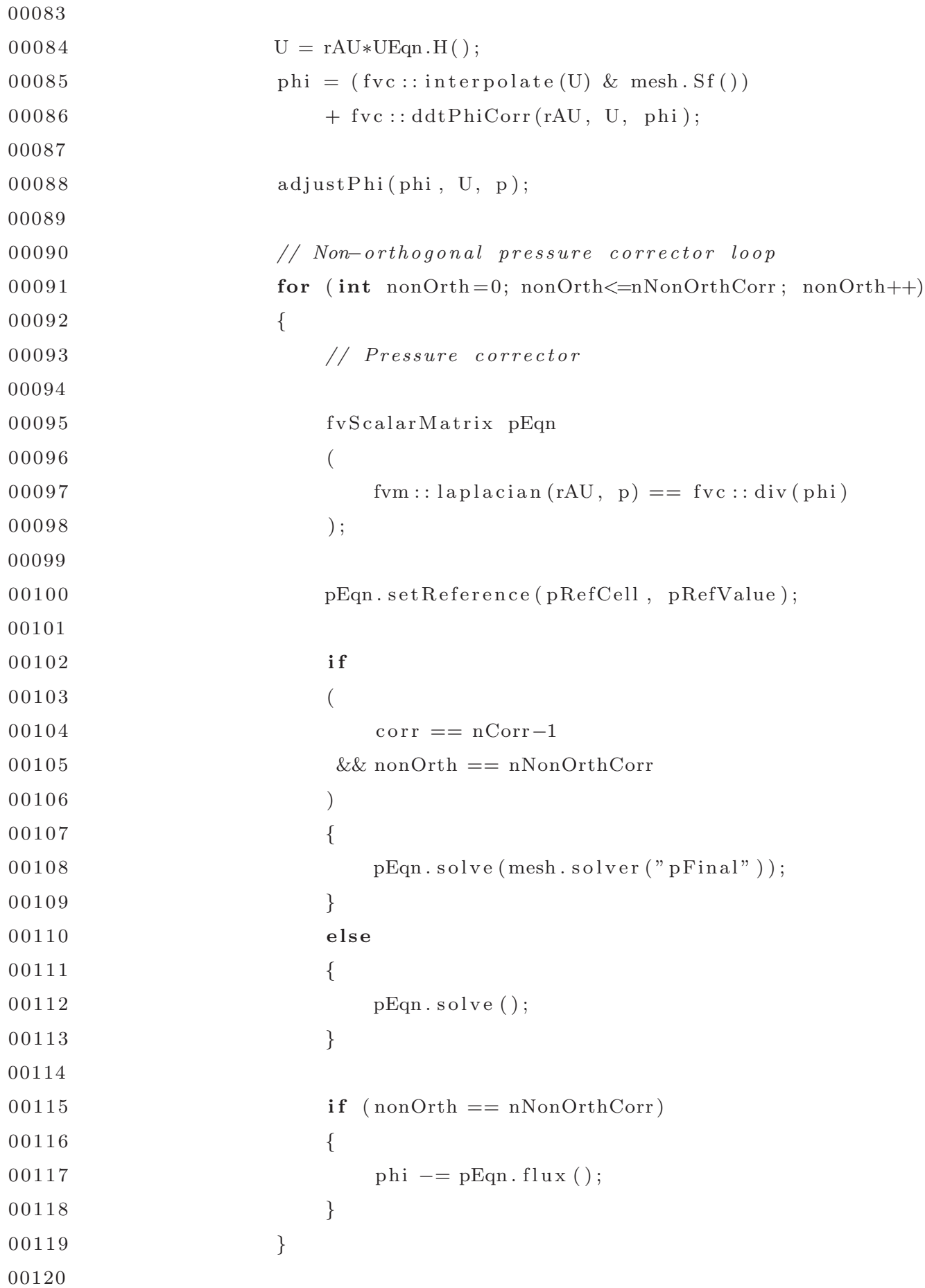




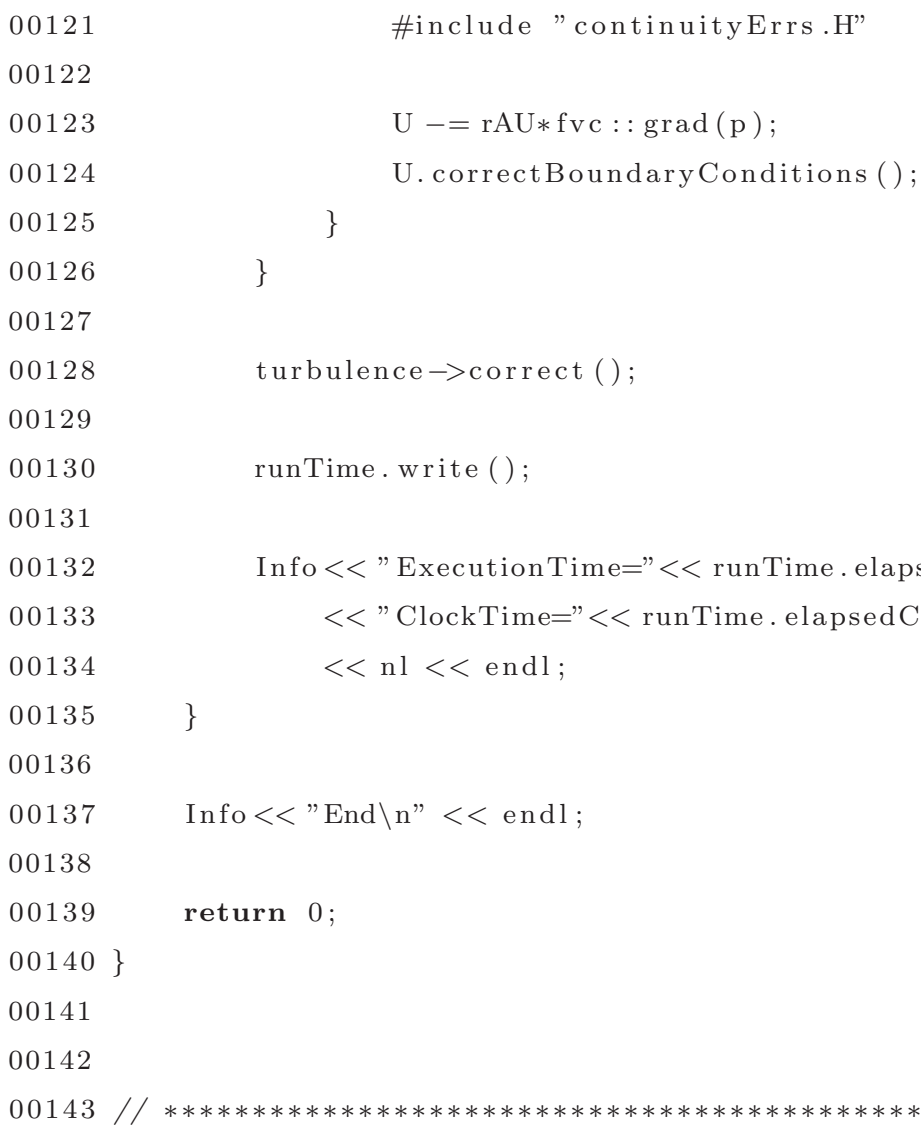

Table 1: Summary of boundary conditions for different cases. $U_{i}$ is the mean velocity and $d / d n$ is a normal derivative to the boundary. The transverse plane is placed at $x_{0}$ where the synthetic turbulence is imposed for XCDF.

\begin{tabular}{llll}
\hline \hline Case & Inlet & Outlet & $x_{0} / \delta=1$ \\
\hline $\mathrm{PBC}$ & $\mathrm{PBC}$ & $\mathrm{PBC}$ & $\mathrm{n} / \mathrm{a}$ \\
$\mathrm{XC}$ & $\mathrm{XC}$ & $d u_{i} / d n=0, p=0$ & $\mathrm{n} / \mathrm{a}$ \\
$\mathrm{XCMC}$ & $\mathrm{XCMC}$ & $d u_{i} / d n=0, p=0$ & $\mathrm{n} / \mathrm{a}$ \\
$\mathrm{XCDF}$ & $u_{i}=U_{i}, d p / d n=0$ & $d u_{i} / d n=0, p=0$ & $\mathrm{XCDF}$ \\
\hline \hline
\end{tabular}




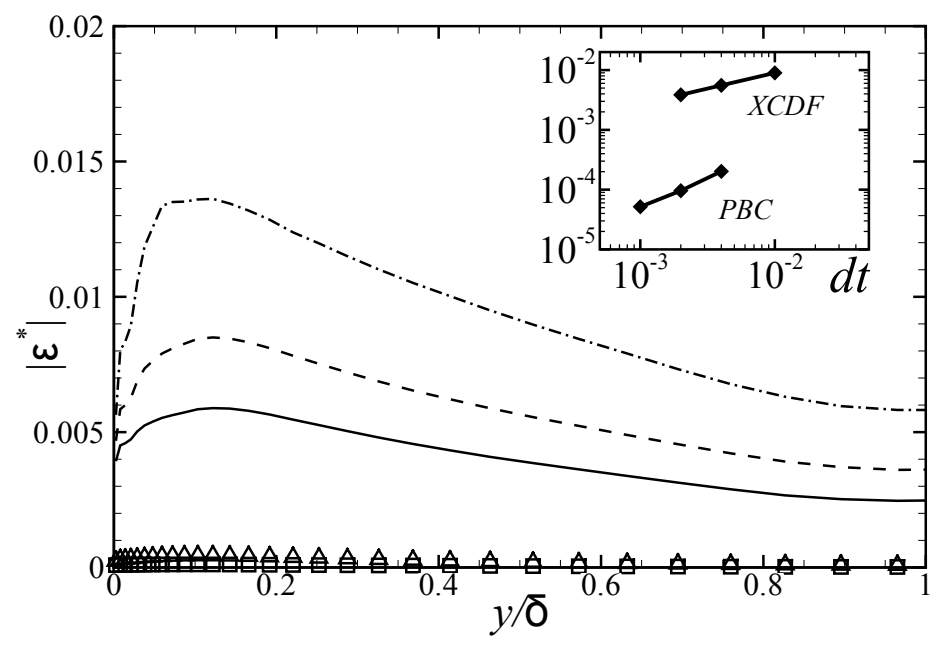

(a)

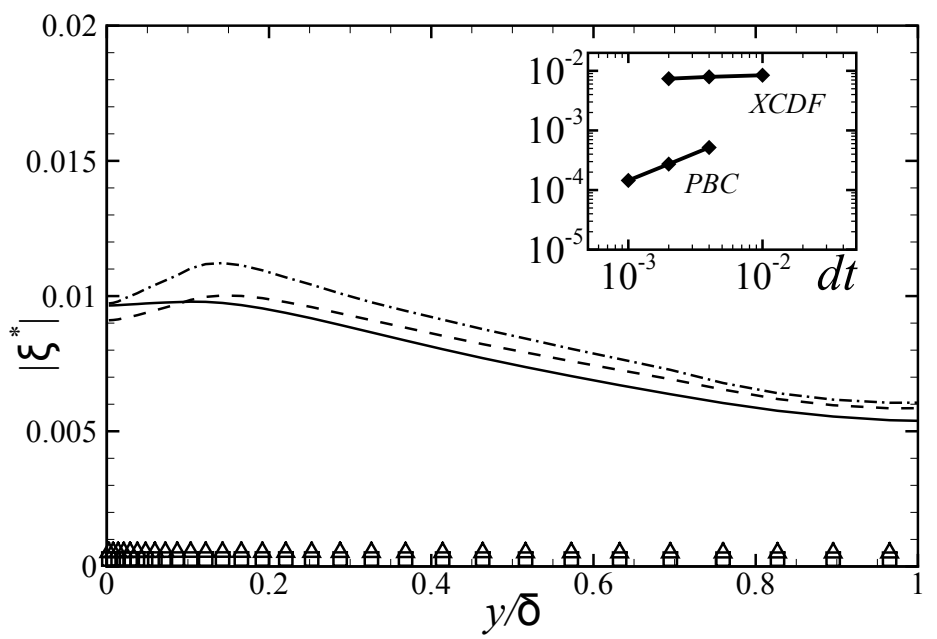

(b)

Figure 1: Profiles of error of (a) the streamwise velocity component, $\left|\varepsilon^{*}\right|$, and (b) pressure, $\left|\xi^{*}\right|$, with different time steps at the plane where synthetic turbulence is imposed, see Eq. 27 for definition. Case PBC: $d t_{*}=0.002 \square, d t_{*}=0.004 \Delta$; XCDF: $d t_{*}=0.002-$, $d t_{*}=0.004--, d t_{*}=0.01-$. where $d t_{*}=d t \times u_{\tau} / \delta$. The insets show the errors against the time step $d t_{*}$ at $y=0.5 \delta$. The errors are normalized appropriately by the bulk mean velocity and density. 


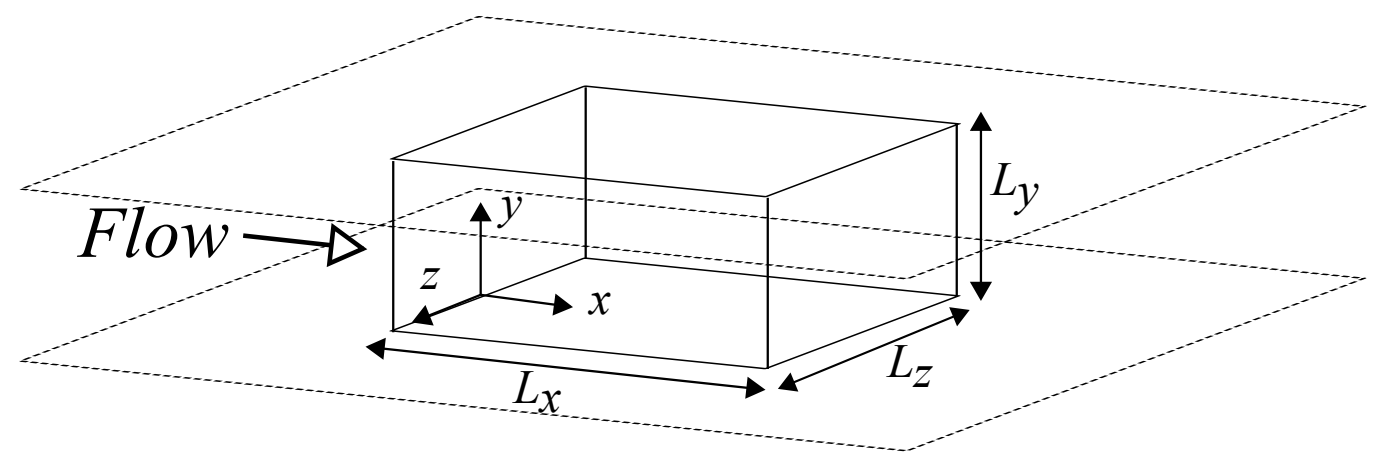

Figure 2: A sketch of the computational domain (not to scale) for a channel flow.

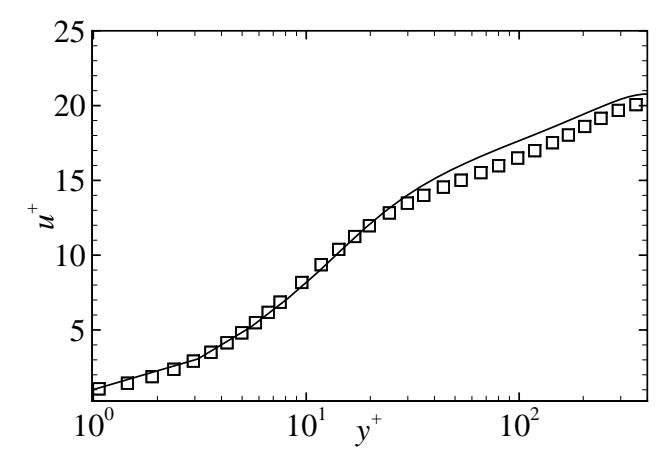

(a)

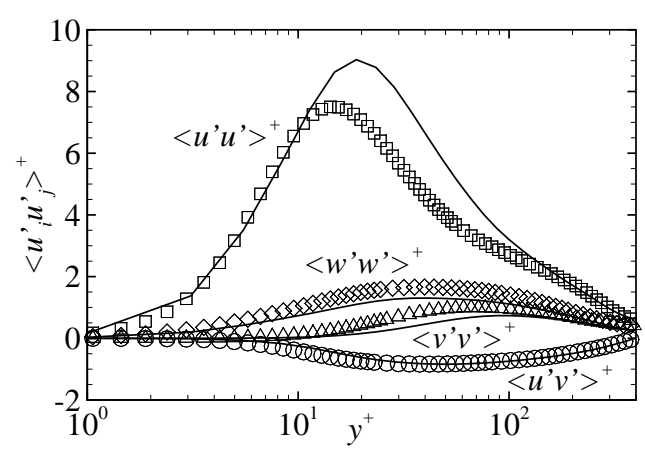

(b)

Figure 3: Profiles of (a) mean velocity and (b) Reynolds stresses from a channel flow. LES results, lines, with the periodic boundary condition are compared with DNS data [10], symbols. Superscript + indicates that the quantities are normalised appropriately by friction velocity $u_{\tau}$, density $\rho$ and dynamic viscosity $\mu$. 


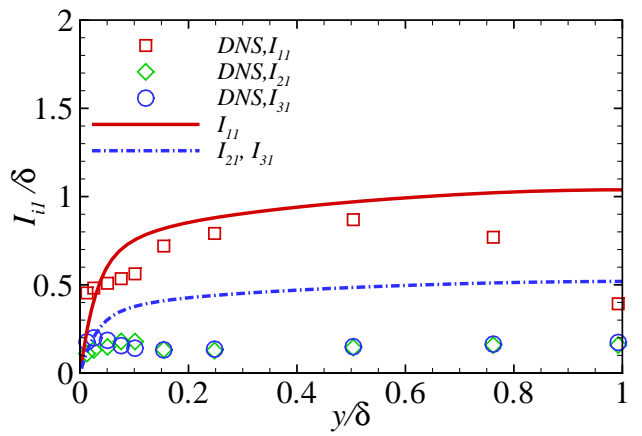

(a)

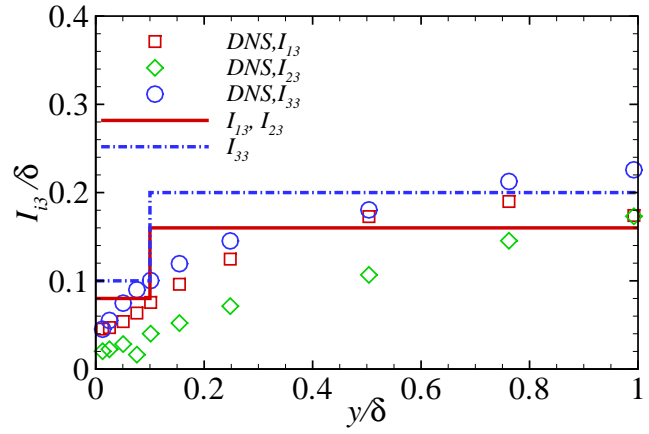

(b)

Figure 4: Integral length scales in (a) the streamwise direction and (b) the spanwise direction (right). Symbols are from DNS [10], lines are specified length scales as input data of the XC, XCMC and XCDF models. The definition of $I_{i j}$ is written in Eq. 7. Note $I_{21}=I_{31}, I_{13}=I_{23}$ and $I_{i 2}=I_{i 3}$.

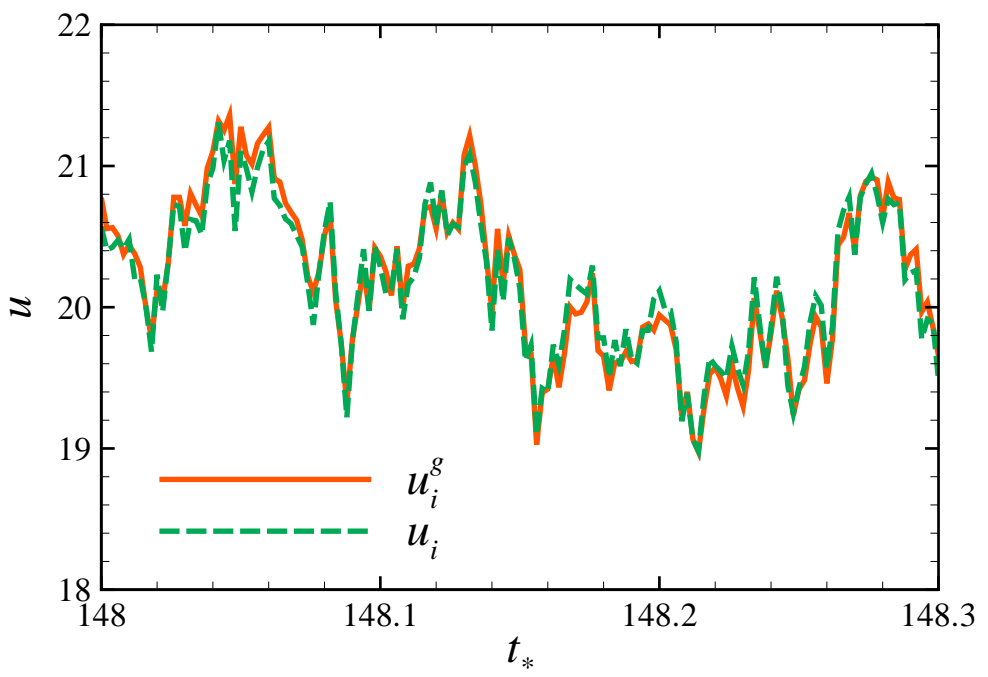

Figure 5: A typical example of the changes of the streamwise velocity before and after the continuity equation (Eq. 15) is satisfied. $u_{i}^{g}$ is the $\mathrm{XC}$ model generated velocity before the continuity equation, and $u_{i}$ is the adjusted velocity after the continuity equation. 


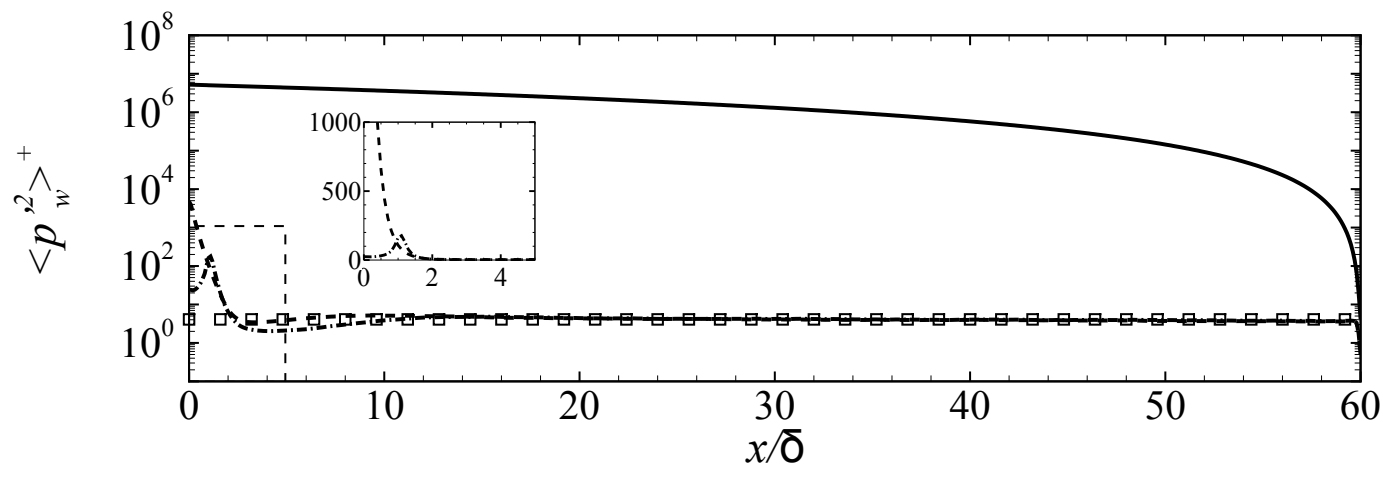

(a)

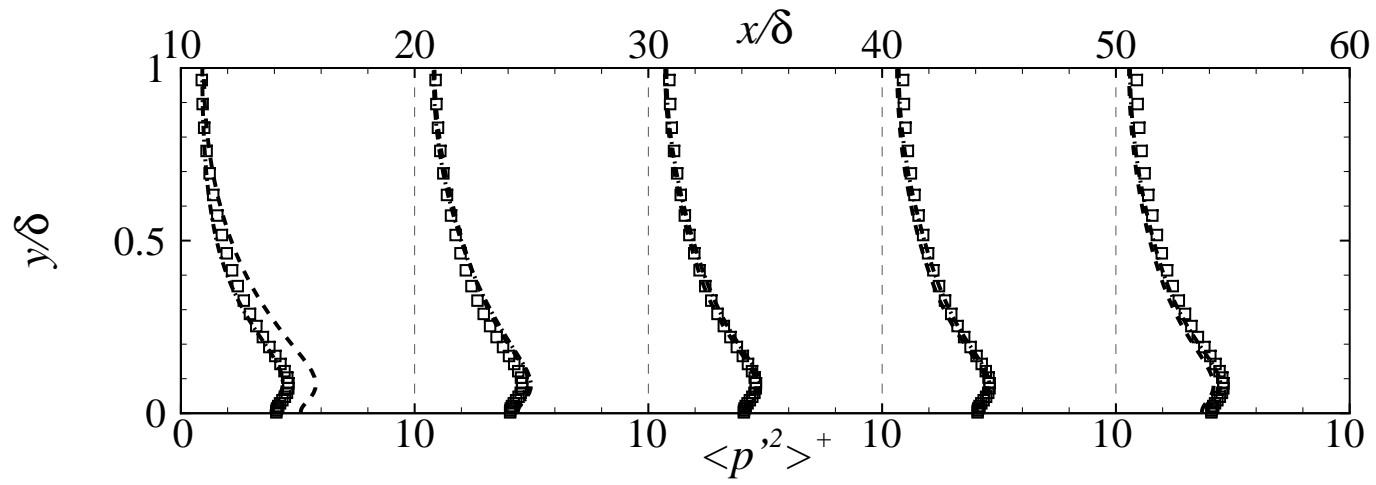

(b)

Figure 6: (a) Development of dimensionless time- and spanwise- averaged variance of the wall pressure fluctuations, $\left\langle p_{w}^{\prime 2}\right\rangle^{+}$. The inset shows a zoomed view of the dashed box on the left bottom corner. (b) Profiles of dimensionless time- and spanwise-averaged variance of pressure fluctuations in the wall-normal direction at the different downstream locations, $<p^{2}>^{+} . \mathrm{PBC} \square, \mathrm{XC}-, \mathrm{XCMC}--, \mathrm{XCDF}-\cdot-$. 


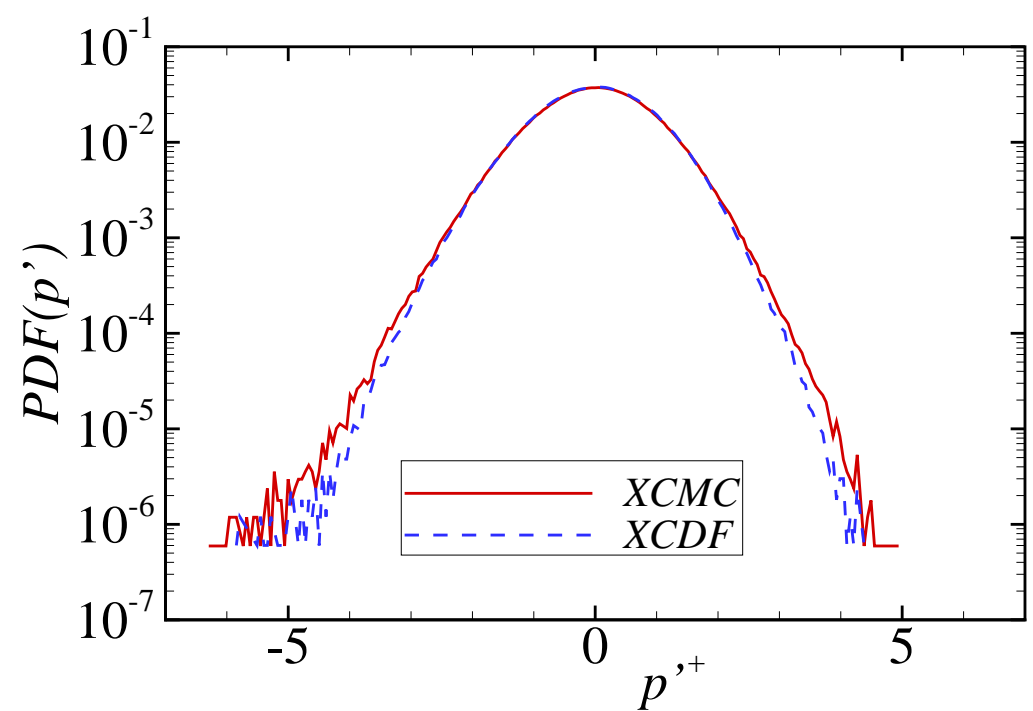

Figure 7: Probability Density Functions (PDFs) of dimensionless pressure fluctuations $p^{\prime+}=p^{\prime} / \rho u_{\tau}^{2}$ sampled at $x / d=5,10,20,30,40,55$ and $y / d=1$. The total number of samples is $2.4 \times 10^{6}$. 


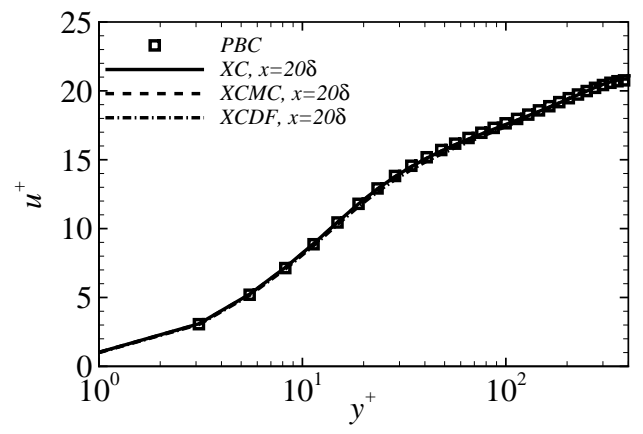

(a)

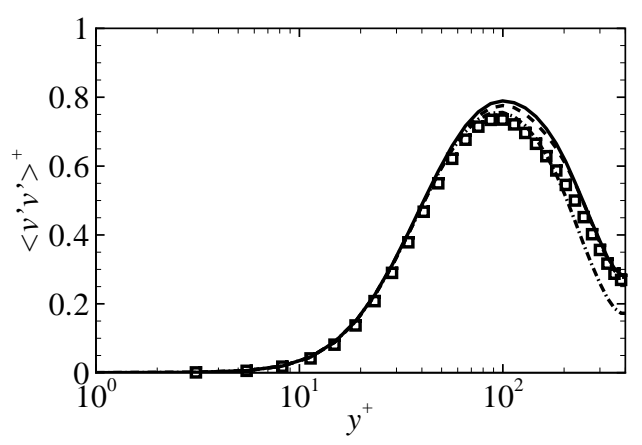

(c)

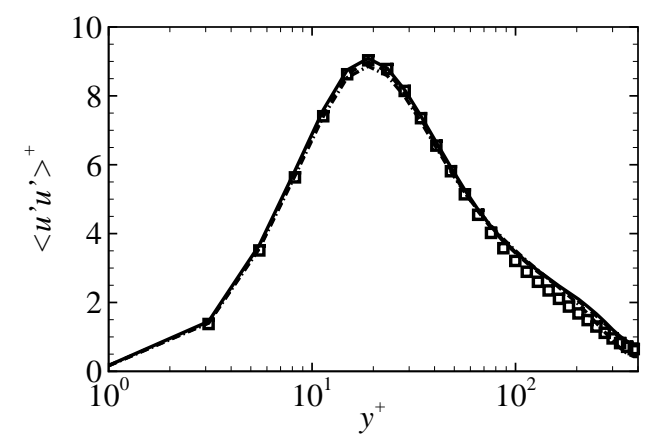

(b)

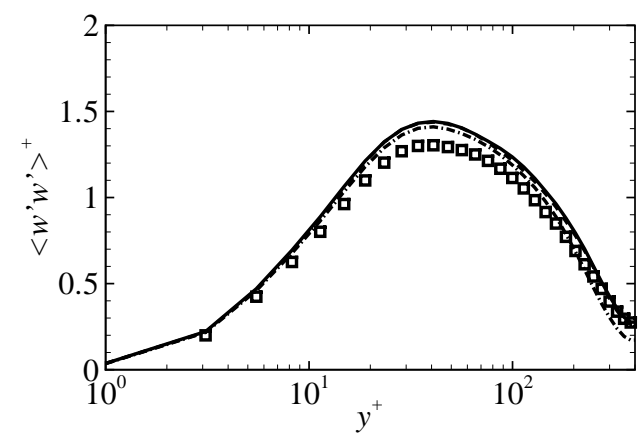

(d)

Figure 8: Profiles of statistics at $x=20 \delta$ obtained from using different inflow methods are compared with those for case PBC. 


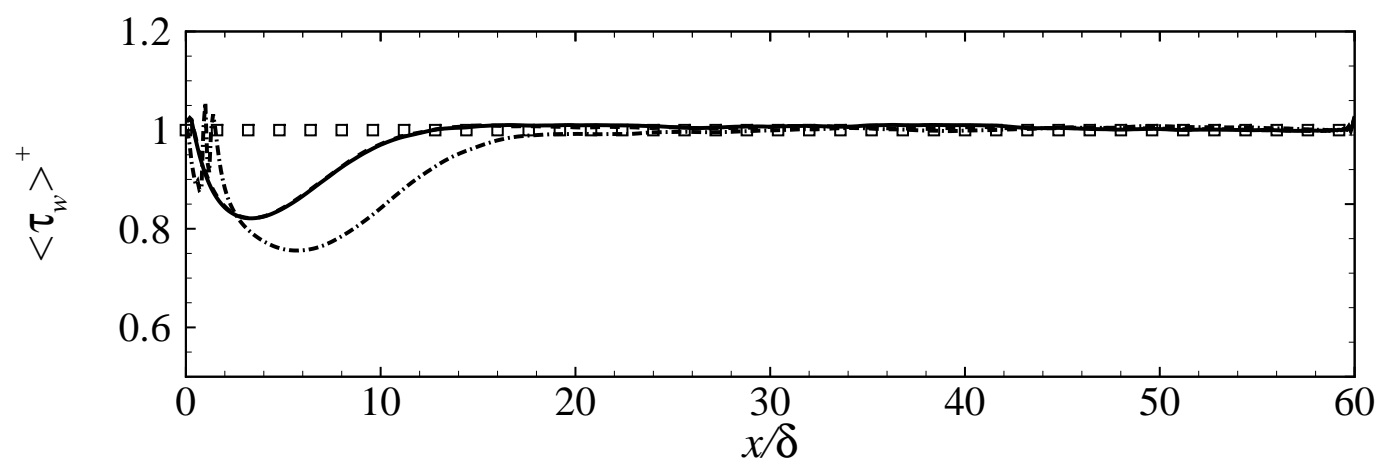

(a)

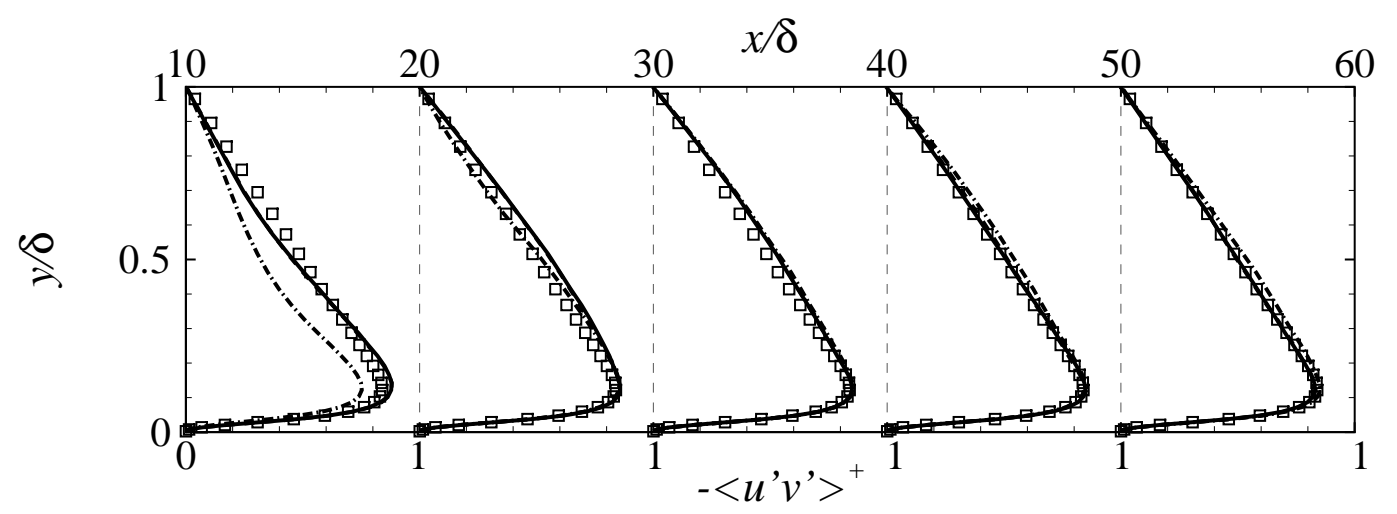

(b)

Figure 9: (a) Development of dimensionless wall shear stress $\tau_{w}^{+}$. (b) Profiles of dimensionless time and spanwise averaged Reynolds shear stress $-\left\langle u^{\prime} v^{\prime}\right\rangle^{+}$at different downstream locations. $\mathrm{PBC} \square, \mathrm{XC}-\mathrm{XCMC}-, \mathrm{XCDF}-\cdot-$. 

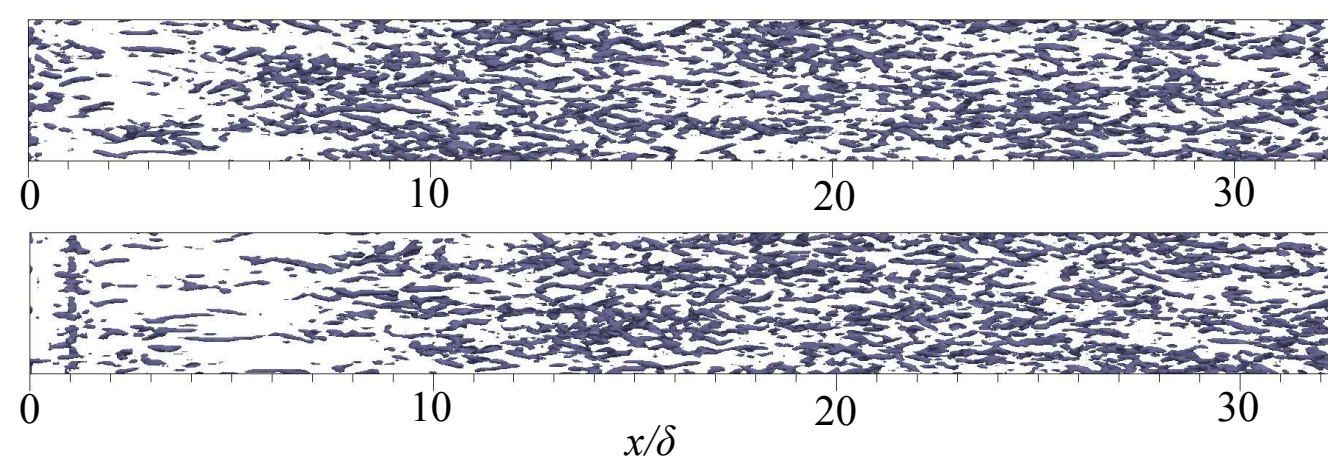

Figure 10: Iso-surface of $Q=200$ in $(x / \delta \leq 32,0<y / \delta<0.25)$. XCMC model(top), and XCDF model(bottom).

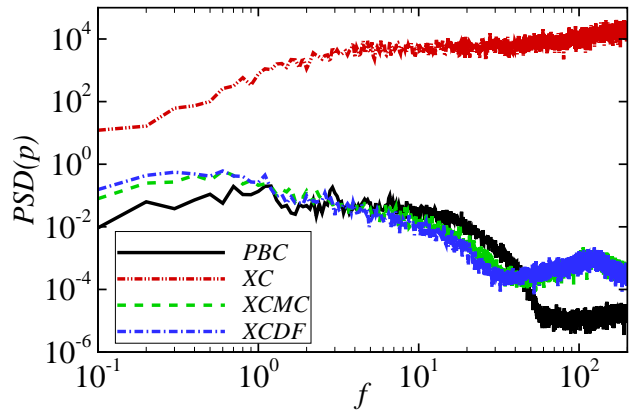

(a)

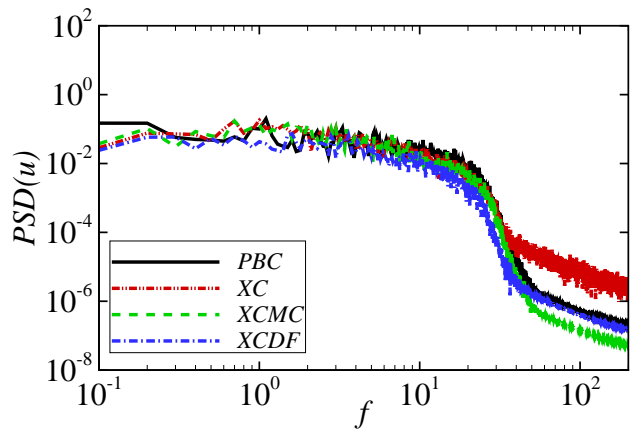

(c)

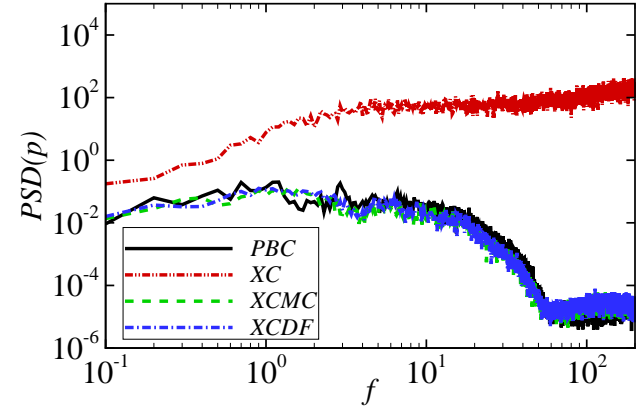

(b)

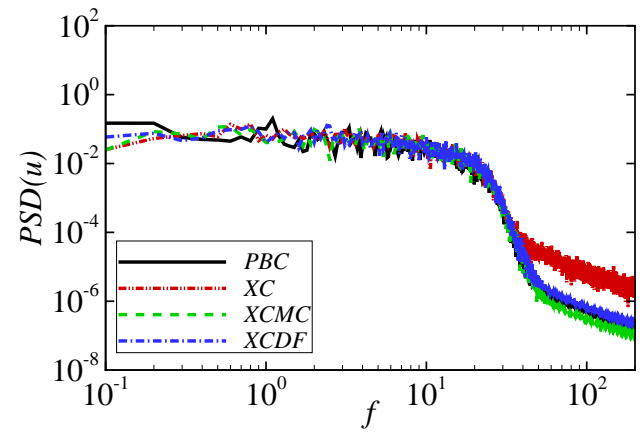

(d)

Figure 11: Power spectral density of pressure fluctuations $(\mathrm{a}, \mathrm{b})$ and the streamwise velocity fluctuations (c,d) at $y / \delta=1$. (a,c) $x / \delta=10 ;(\mathrm{b}, \mathrm{d}) x / \delta=55$. All quantities are normalized appropriately by $u_{\tau}$ and $\delta$. 